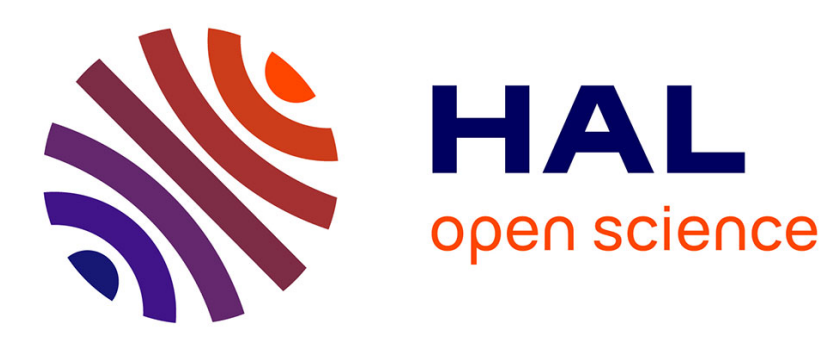

\title{
A nonlinear Domain Decomposition formulation with application to granular dynamics
}

Pierre Alart, Damien Iceta, David Dureisseix

\section{To cite this version:}

Pierre Alart, Damien Iceta, David Dureisseix. A nonlinear Domain Decomposition formulation with application to granular dynamics. Computer Methods in Applied Mechanics and Engineering, 2012, 205-208, pp.59-67. 10.1016/j.cma.2011.04.024 . hal-00597519

\section{HAL Id: hal-00597519 https://hal.science/hal-00597519}

Submitted on 6 Jun 2011

HAL is a multi-disciplinary open access archive for the deposit and dissemination of scientific research documents, whether they are published or not. The documents may come from teaching and research institutions in France or abroad, or from public or private research centers.
L'archive ouverte pluridisciplinaire HAL, est destinée au dépôt et à la diffusion de documents scientifiques de niveau recherche, publiés ou non, émanant des établissements d'enseignement et de recherche français ou étrangers, des laboratoires publics ou privés. 


\title{
A nonlinear Domain Decomposition formulation with application to granular dynamics
}

\author{
P. Alart, D. Iceta, D. Dureisseix \\ Laboratoire de Mécanique et Génie Civil (LMGC), University Montpellier 2 / CNRS UMR5508 CC048, Place E. \\ Bataillon, F-34095 MONTPELLIER CEDEX 5, France
}

\begin{abstract}
Simulation of granular media undergoing dynamic evolution involves nonsmooth problems when grains are modeled as rigid bodies. With dense samples, this nonsmoothness occurs everywhere in the studied domain, and large sized systems lead to computationally intensive simulations. In this article, we combine domain decomposition approaches and nonsmooth contact dynamics. Unlike the smooth continuum media case, a coarse space problem does not trivially increase the convergence rate, as it is exemplified in this article, with semi-analytical examples and real size numerical simulations. Nevertheless, the description of an underlying force network in the samples may guide the analysis for new approximation schemes or algorithms.
\end{abstract}

Keywords: Nonsmooth contact dynamics, multicontact systems, scalability, multiscale, asymptotic analysis

2000 MSC: 65Y05, 70E55, 76T25

\section{Introduction}

To underline the objectives of this paper it is first important to consider associated methods at the crossroads of two fields: the NonSmooth Contact Dynamics (NSCD) and the Domain Decomposition Methods (DDM). These two approaches are combined here to the limits of their possibilities. NSCD or Contact Dynamics in short, has been developed by J. J. Moreau and M. Jean over the last two decades $[1,2]$. It is suited for many applications but has proven to be particularly suitable when collections of rigid or deformable bodies are packed together in a dense assembly and subject to dynamic loading deformations. Moreover, much attention has been paid to the way the method converges and how to access the quality of a numerical simulation, which is a difficult issue when dealing with large collections of bodies for which the number and nature

Email address: \{pierre.alart, damien.iceta,david.dureisseix\}@univ-montp2.fr (P. Alart, D. Iceta, D. Dureisseix) 
of the solutions are far from being unique. Numerical simulations thus have to be performed using a fully implicit resolution of the contact forces. At each step of the evolution problem, all kinematic constraints within the packing are simultaneously taken into account along with the equations of motion to determine all contact forces. This allows us to deal properly with nonlocal momentum transfers involved in multiple collisions, contrary to classical molecular dynamics schemes that consider the system evolution as a succession of binary collisions [3]. The computational cost may be quite high, but the gain is substantial. For instance in granular materials, commonly accepted ideas about distributions of stress or strain in continuous media are no longer true. Chains of forces act between grains in two ways i.e. the strong force network supports shear stress while the weak force network contributes to internal pressure [4]. Simulations of very large granular systems can range from the study of $10 \mathrm{~m}$ of railway ballast submitted to cyclic dynamic loading [5], to the behavior of the Nîmes arena and Arles aqueduct (France) subjected to seismic loading [6], which are examples of two challenges in computational mechanics. The efficiency of the Domain Decomposition methods in the context of multiprocessor computations is well established from theoretical and practical standpoints when dealing with a linear system derived from a discretization of a continuous problem [7, 8]. The scalability may be proved theoretically when a coarse problem is added to the preconditioner of a conjugate gradient algorithm applied to the interface problem [9]. Enrichment of the coarse problem turns out to be mandatory to improve the convergence for different situations such as 3D [10], plate bending [11] or dynamic problems [12]. In [13] a multilevel domain decomposition technique is used as a numerical strategy to simulate the behavior of nonsmooth discrete media and to provide the macroscopic numerical behavior of the same system. Analogously to the "corners" or "wedges" of 3D continuous problems, the emergence of weak interfaces, i.e. characteristics of discrete media sub-structuring, is taken into account in the coarse space. However, the study is restricted to quasi-static simulation of a large-scale tensegrity grid. Dynamic modeling of a granular medium is quite different as we will see later. Moreover, extension of DDM to nonlinear problems is commonly tackled with a resolution of a series of linear problems, with each of them being solved with a classical DD solver as a black box [14, 15] and few attempts to tackle nonlinearity and domain decomposition in a one shot algorithm [16]. Such a strategy is difficult to efficiently extend to granular type problems. When the contact may only be accounted for at the interface of the subdomains it is possible to extend sophisticated linear domain decomposition solvers to these cases $[17,18,19]$. Nevertheless such a strategy is not feasible for granular type problems. The main objective is now to investigate discrete multi-large-scale dynamic systems. A domain decomposition strategy may provide both an efficient solver and a numerical homogenized model directly derived from the simulation. This continuous homogenized model could be substituted for the discrete one in some parts of the domain as long as these parts continue to evolve in a smooth enough way that they can be determined. This latter issue is nevertheless beyond the scope of this paper as for the sub-structuring technique adapted to discrete systems with permanent evolution of the connectivity of the particles, which is characteristic of granular flows.

For general purposes, we consider a model problem involving a dynamically loaded discrete system with nonlinear or nonsmooth interactions between their components. In line with the NSCD approach, we opt for the velocity-impulse formulation presented in the second section together with a multidomain reformulation and generic algorithm. The multidomain reformulation is based on a FETI-type approach where the subdomains are "glued" by Lagrange multipliers which are inter-domain forces. This choice is in accordance with the NSCD approach where impulses are privileged variables. The generic solving method consists of a two-stage Gauss-Seidel 
type algorithm. Such a strategy recovers the generic NonLinear Gauss-Seidel (NLGS) method applied in conjunction with the NSCD formulation; for details about convergence, refer to [20]. The following developments have three main objectives. The first one consists in proposing an improved algorithm combining the advantages of the NLGS and LATIN (for LArge Time INcrement) methods [21, 22] previously compared in [23]. The improvement is motivated by features of the interface problem derived from the FETI-type approach. The associated operator turns out to be quasi-diagonal such that no long distance interactions are generated and no macro quantities can be extracted from this interface problem. The second objective is therefore to enhance the interface problem so as to accelerate the convergence and define a possibly macro-homogenized model. Finally, the role of the rigid body motions in the convergence of the algorithm has to be clarified. Indeed the insertion of rigid body motions in the coarse problem is essential for scalability in elastostatics; this has been theoretically proved. It is thus necessary, from a numerical standpoint, to improve the convergence in linear elastodynamics [12], as a better preconditioner. An asymptotic analysis is dedicated to this open question for a collection of rigid bodies with contact interactions. As it is hard to derive analytical results at the end of this analysis, some small examples using MAPLE ${ }^{\mathrm{TM}}$ software will be investigated. Using such simple examples to investigate complex problems resembles the approach developed by J.A.C. Martins for studying, for example, the instability of systems involving friction [24]. J.A.C. Martins studied mechanical behavior whereas we study numerical behavior.

\section{Fundamental equations and reference problem}

The following terminology will be used throughout this article:

\begin{tabular}{ll}
\hline$V$ & velocity fields \\
$R$ & impulse fields \\
$M$ & inertial operator \\
$R^{d}$ & prescribed impulses fields \\
$v$ & kinematic material variable \\
$r$ & sthenic material variable \\
$H^{T}$ & linear operator between $V$ and $v$ \\
\hline
\end{tabular}

Sthenics is the science of the efforts as the kinematics is the science of the motions. The common expression is "static variables".

A time-stepping scheme is used, where quantities at the beginning of the time slab with a superscript (-) are known, and quantities at the end of the time slab without a superscript have to be determined.

The dynamics of the granular medium is written as the following vector equation [2], where the matrix $M$ is diagonal, provided that the global coordinates of rotation vectors are expressed in the inertia eigenbasis of each grain:

$$
M V-R=F^{d}
$$

where the prescribed right hand side is $F^{d}=R^{d}+M V^{-}$.

Here we focus on simple unilateral contact which is naturally expressed as a complementary condition linking contact force to a gap. For dynamics, Moreau proved via a viability lemma [2], that we can use a velocity-impulse complementary law. The constitutive relation is summarized in the following formal equation:

$$
\mathcal{R}(v, r)=0
$$




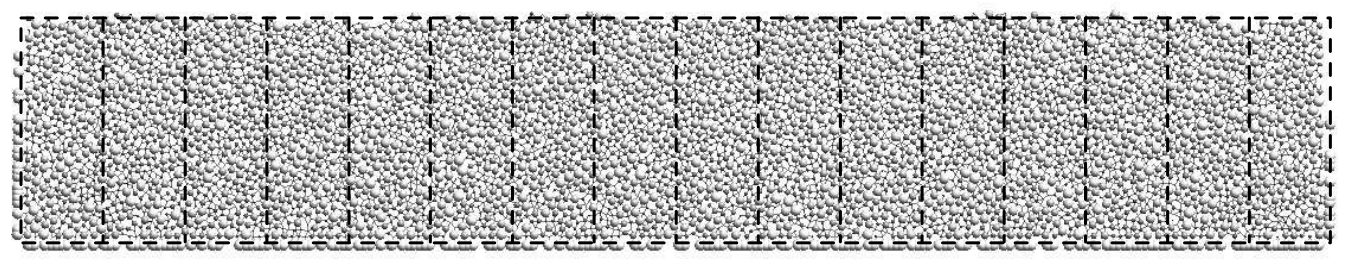

Figure 1: Geometrical partitioning of the domain

Finally, the compatibility conditions related to the connectivity of the links or contacts, read:

$$
v=H^{T} V \quad \text { and } \quad R=H r
$$

Taking the dynamics (1) and the compatibility conditions (3) into account, the reduced dynamics involving material variables can be obtained:

$$
W r-v=-v^{d}
$$

where $W$ is the Delassus operator: $W=H^{T} M^{-1} H$, and $v^{d}=H^{T} M^{-1} F^{d}$.

To close the problem, one adds the constitutive relation (2), and the reference problem reads:

$$
\left\{\begin{array}{l}
W r-v=-v^{d} \\
\mathcal{R}(v, r)=0
\end{array}\right.
$$

\section{Multidomain reformulation and associated algorithm}

The domain has to be split into subdomains in order to use parallelization for the solving phase. This decomposition is performed at each time step for accounting for the migration of grains from one subdomain to another. Such a strategy may be implemented with minimal computational efforts using sophisticated routines out of the purpose of this paper. Since the nonsmoothness may occur in interactions between grains, we choose to distribute interactions among subdomains as in [23] (we proceed by distributing the middle points between the centers of mass of interacting grains, according to their coordinates, using an arbitrary regular underlying grid, Figure 1). Indeed, with such a choice, the "boundary" grains are split between two subdomains. For consistency, the masses and moments of inertia are distributed among the neighboring subdomains according to their number. The interface between two subdomains is defined as the set of these grains, that joins the subdomains. The nonsmoothness is therefore localized only within the subdomains. This modeling choice is identical to [15] and somehow the dual of that proposed in [21], where nonlinearities (contact on crack lips) are isolated at the interfaces.

In each subdomain $E$, the reference problem is identical to the global one (with the subscript $E$ ). On the global interface noted $\Gamma$ an interconnecting condition (on velocities of boundary grains) has been added to "glue" neighboring subdomains, where $A_{E \Gamma}$ is a signed boolean matrix:

$$
\sum_{E=1}^{n_{s d}} A_{E \Gamma} V_{E}=0
$$


Formally the previous summation is performed on all the subdomains (number equal to $n_{s d}$ ), even if, for a given interface grain the only neighboring subdomains have to be considered. Then we obtain a FETI-like formulation for the reference problem using a multiplier field $F_{\Gamma}$ and the notations $\hat{A}_{E \Gamma}^{T}=H_{E}^{T} M_{E}^{-1} A_{E \Gamma}^{T}$ :

$$
\begin{aligned}
& \left.\begin{array}{l}
W_{E} r_{E}-v_{E}-\hat{A}_{E \Gamma}^{T} F_{\Gamma}=-v_{E}^{d} \\
\mathcal{R}\left(v_{E}, r_{E}\right)=0 \\
\sum_{E=1}^{n_{s d}} A_{E \Gamma} V_{E}=0
\end{array}\right\} E=1, \ldots, n_{s d}
\end{aligned}
$$

The reduced problem on $\left(r_{E}, v_{E}, F_{\Gamma}\right)$, with the notations $\hat{f}=\sum_{E} A_{E \Gamma} M_{E}^{-1} F_{E}^{d}$ and $X=\sum_{E} A_{E \Gamma} M_{E}^{-1} A_{E \Gamma}^{T}$, reads:

$$
\begin{aligned}
& \left.\begin{array}{l}
W_{E} r_{E}-v_{E}-\hat{A}_{E \Gamma}^{T} F_{\Gamma}=-v_{E}^{d} \\
\mathcal{R}\left(v_{E}, r_{E}\right)=0 \\
X F_{\Gamma}-\sum_{E=1}^{n_{s d}} \hat{A}_{E \Gamma} r_{E}=\hat{f}
\end{array}\right\} E=1, \ldots, n_{s d}
\end{aligned}
$$

The associated algorithmic formulation is described in algorithm 1 , where $N$ is the number of time steps and $n_{\mathrm{GS}}$ is the prescribed number of Gauss-Seidel iterations to solve the nonsmooth dynamics per subdomain.

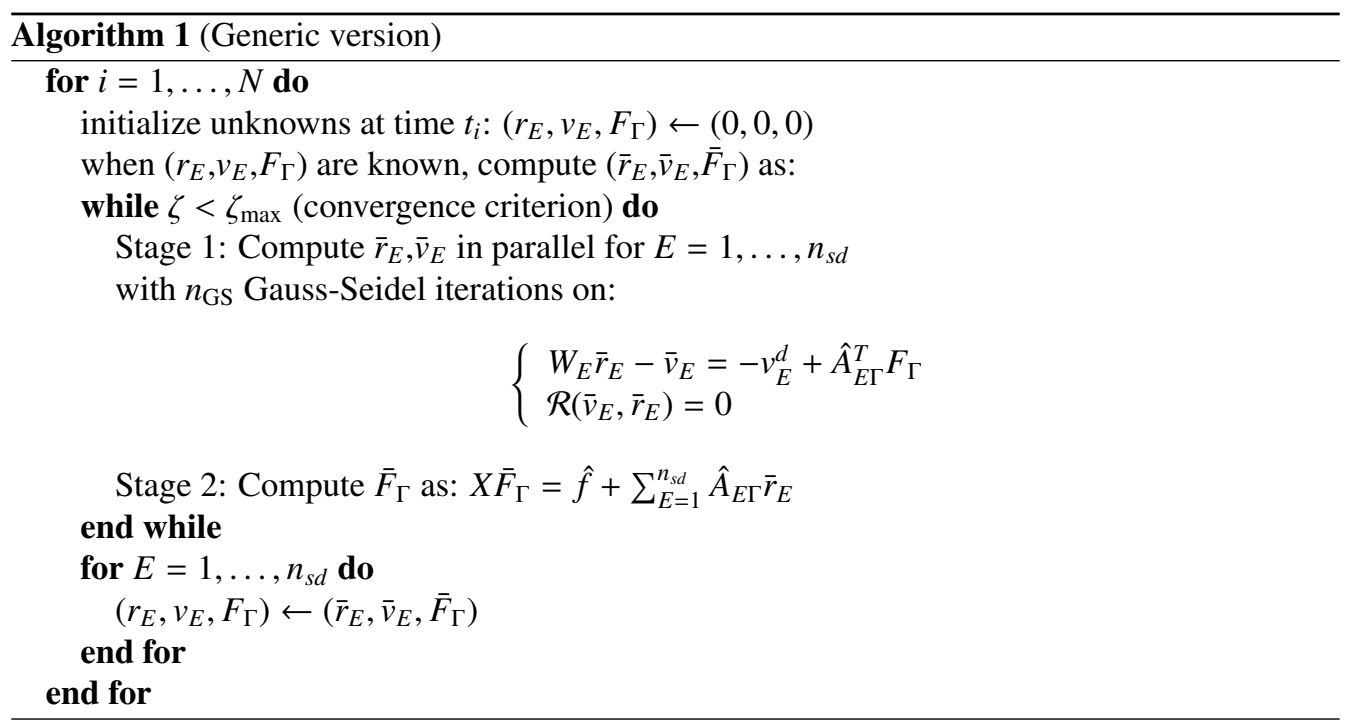

The convergence of such an algorithm involving nonsmooth interaction laws between grains is very difficult to investigate from a mathematical point of view. Even the convergence of the Gauss-Seidel method embedded in the Stage 1 can be only proved for special cases [20]. The following study consists in investigating first, and modestly, the convergence rate of the global algorithm with linear interaction laws and in extracting some conclusions. 


\subsection{Convergence analysis for the linear case}

A linear problem is characterized by a linear interaction law: the only adaptation to the reference problem is the constitutive relation (2), which now reads: $r=-k v$, where $k$ is an integral viscosity parameter. It is convenient in the following to consider a flexibility-like parameter $\epsilon$, which is identical for all the interactions, such that:

$$
\mathcal{R}\left(\bar{v}_{E}, \bar{r}_{E}\right)=0 \Leftrightarrow \bar{r}_{E}=-k_{E} \bar{v}_{E}=-\frac{1}{\epsilon} \bar{v}_{E}
$$

By eliminating local variables $\left(\bar{r}_{E}, \bar{v}_{E}\right)$ in the first stage of algorithm 1 , we can define a fixed point algorithm applied to the interface multiplier $F_{\Gamma}$. The contraction matrix then depends on the flexibility parameter as follows:

$$
B_{\epsilon}=X^{-1} Y_{\epsilon}
$$

where

$$
Y_{\epsilon}=\sum_{E=1}^{n_{s d}} \hat{A}_{E \Gamma} W_{E, \epsilon}^{-1} \hat{A}_{E \Gamma}^{T} \quad \text { and } \quad W_{E, \epsilon}=W_{E}+\epsilon I
$$

A theoretical estimation of the spectral radius of this matrix is not available, but a numerical evaluation may be performed for simple examples in order to compare the performance of this generic algorithm with a forthcoming one. The first simple example consists of a $2 \mathrm{D}$ frictionless granular problem with 4 disks and potentially 5 contacts, which are represented with solid and dashed lines in Figure 2. (The difference between solid and dashed line is explained in a next section for further studies.) A solution is given on the same figure for a prescribed velocity on one of the disks. Note that some tensions are non-negative because the loss of contact is not taken into account in this example. This system is split into two subdomains: the first one contains one disk and two half-disks, while the second one contains one disk and the two complementary half-disks. Two more complex examples are depicted in Figures 3 and 4. The spectral radius of the contraction matrix is given with respect to $\epsilon$ in Figure 5; two special values are distinguished: $\epsilon=1$ and the limit case $\epsilon=0$.

\section{Global behavior and enriched algorithm}

\subsection{General case}

For dense granular systems, the dynamic equations alone are not able to reproduce the collective (i.e. global) behavior of the sample. Now the previous global operator $X$ only involves the dynamics via the inertia matrices $M_{E}$; this operator is then diagonal and therefore cannot represent any information transfer between grains with a long-distance correlation through subdomains. Interactions between grains must be considered when introducing a collective behavior at the second stage of the algorithm. We thus propose to introduce an additional search direction in the previous algorithm 1, while not changing the reference problem, which can be interpreted as a numerically tangent constitutive behavior between grains. Indeed such a search direction is similar to the procedure introduced in the LATIN method [21] or in the Uzawa correction of the Augmented Lagrangian approach. It therefore introduces an additional parameter that will have to be discussed and chosen. 


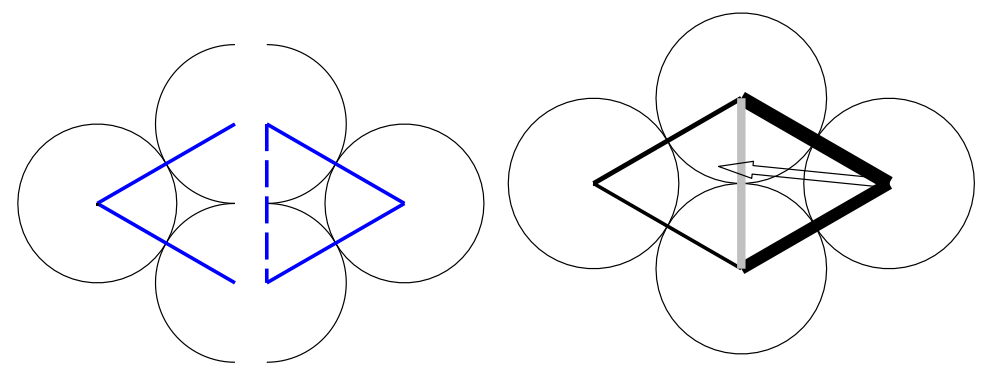

Figure 2: A first example with 4 disks and 5 links split into two subdomains and a resulting tension network (black for compression, grey for tension) for a given velocity of the grain on the right (doubled arrow)
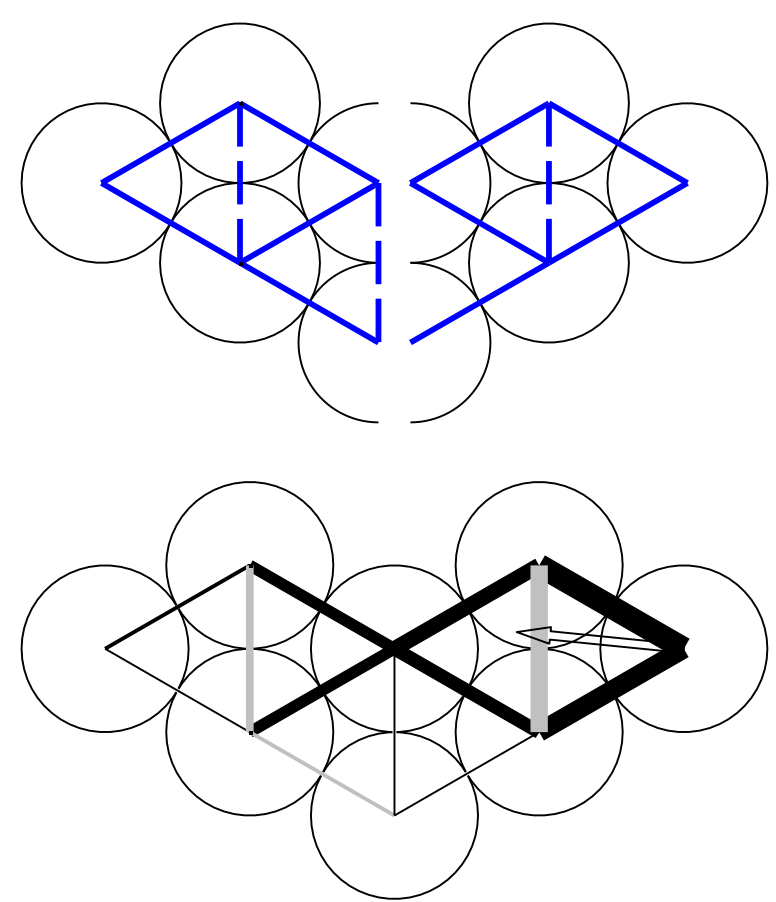

Figure 3: A second example with 8 disks and 13 links split into two subdomains and a resulting tension network (black for compression, grey for tension) for a given velocity of the grain on the right (doubled arrow) 

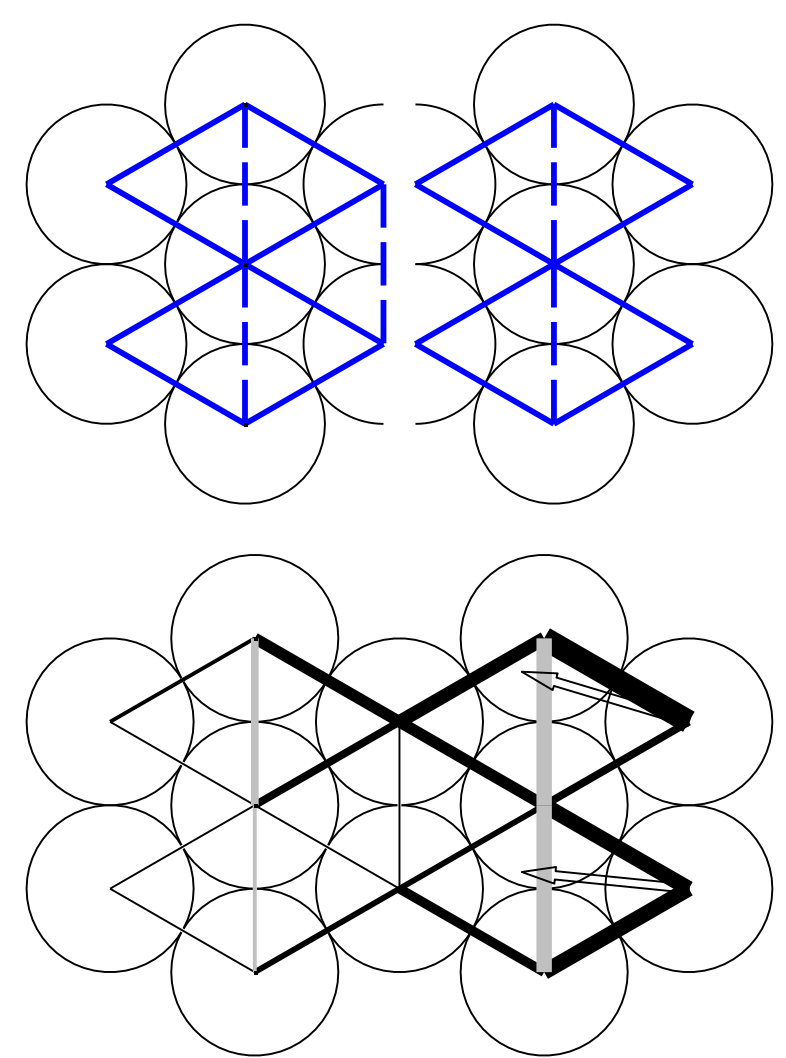

Figure 4: A third example with 12 disks and 21 links split into two subdomains and a resulting tension network (black for compression, grey for tension) for a given velocity of the grains on the right (doubled arrow) 


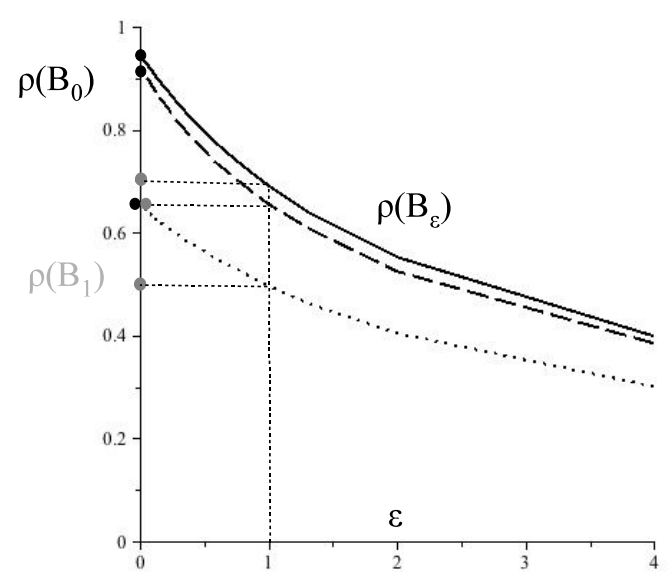

Figure 5: Evolution of the spectral radius vs. $\epsilon$ for the three examples: 4 disks (dot), 8 disks (dash), 12 disks (solid)

The previous algorithm is therefore modified as follows. The first stage still consists of finding the material quantities $\left(\bar{v}_{E}, \bar{r}_{E}\right)$ satisfying:

$$
\begin{aligned}
W_{E} \bar{r}_{E}-\bar{v}_{E} & =\hat{A}_{E \Gamma} F_{\Gamma}-v_{E}^{d} \\
\mathcal{R}\left(\bar{v}_{E}, \bar{r}_{E}\right) & =0
\end{aligned}
$$

which is not dependent on each subdomain $E=1, \ldots, n_{s d}$.

The second stage is modified. It requires to find $\left(\tilde{v}_{E}, \tilde{r}_{E}\right)$ and $F_{\Gamma}$, once $\left(\bar{v}_{E}, \bar{r}_{E}\right)$ are known from the previous stage, satisfying:

- the dynamic equations,

- the interface continuity constraint,

- the new search direction $\left(\tilde{r}_{E}-\bar{r}_{E}\right)+l_{E}\left(\tilde{v}_{E}-\bar{v}_{E}\right)=0$.

$l_{E}$ is an additional numerical parameter in the algorithm. As for $k_{E}$ it is convenient for the forthcoming analysis to introduce a parameter that is identical for all interactions: $l_{E}=\frac{1}{\eta}$. When introducing the grain velocity field $\tilde{V}_{E}$ at this stage, the dynamics reads

$$
M_{E} \tilde{V}_{E}+A_{E \Gamma}^{T} F_{\Gamma}=F_{E}^{d}+H_{E} \tilde{r}_{E}
$$

Substituting $\tilde{r}_{E}$ with the new search direction, using the kinematical admissibility $\tilde{v}_{E}=$ $H_{E}^{T} \tilde{V}_{E}$, gives

$$
M_{\eta, E} \tilde{V}_{E}=F_{E}^{d}+H_{E}\left(\bar{r}_{E}+l_{E} \bar{v}_{E}\right)-A_{E \Gamma}^{T} F_{\Gamma}
$$

with $M_{\eta, E}=M_{E}+L_{E}$ and $L_{E}=H_{E} l_{E} H_{E}^{T}$ which contains informations on the interaction network thanks to the connectivity matrix $H_{E}$. As previously, the dynamic equations are condensed onto the interforces, and the continuity constraints at the interfaces gives the new global behavior with $\bar{F}_{\Gamma}$ as unknown:

$$
X_{\eta} \bar{F}_{\Gamma}=\sum_{E=1}^{n_{s d}} A_{E \Gamma} M_{\eta, E}^{-1}\left[F_{E}^{d}+H_{E}\left(\bar{r}_{E}+l_{E} \bar{v}_{E}\right)\right]
$$


with the new global interforce operator

$$
X_{\eta}=\sum_{E=1}^{n_{s d}} A_{E \Gamma} M_{\eta, E}^{-1} A_{E \Gamma}^{T}
$$

This constitutes the modified second stage of the algorithm.

\subsection{Linear case and convergence analysis} that

In such a case, the first stage simplifies into: find $\bar{r}_{E}$ for each subdomain independently such

$$
\left(W_{E}+k_{E}^{-1}\right) \bar{r}_{E}=-v_{E}^{d}+\hat{A}_{E \Gamma}^{T} F_{\Gamma}
$$

$k_{E}$ is a diagonal matrix with entries $k$ for each interaction.

For the second stage, the right hand side also simplifies, since

$$
\bar{r}_{E}+l_{E} \bar{v}_{E}=\left(1-l_{E} k_{E}^{-1}\right) \bar{r}_{E}
$$

As for the generic method, the local variables may be eliminated to obtain a fixed point algorithm for which the contraction matrix is quite complicated and depends both on the physical and numerical flexibility parameters: $\epsilon$ and the additional parameter $\eta=\frac{1}{l_{E}}$, as:

$$
B_{\epsilon, \eta}=X_{\eta}^{-1} Y_{\epsilon, \eta}
$$

where

$$
\begin{aligned}
X_{\eta} & =\sum_{E=1}^{n_{s d}} A_{E \Gamma} M_{E, \eta}^{-1} A_{E \Gamma}^{T} \\
Y_{\epsilon, \eta} & =\sum_{E=1}^{n_{s d}} A_{E \Gamma} M_{E, \eta}^{-1} H_{E}\left(1-\frac{\epsilon}{\eta}\right) W_{E, \epsilon}^{-1} \hat{A}_{E \Gamma}^{T} \\
M_{E, \eta} & =M_{E}+\frac{1}{\eta} K_{E} \\
K_{E} & =H_{E} H_{E}^{T}
\end{aligned}
$$

When $\eta=\epsilon$ the matrix $Y_{\epsilon, \eta}$ is obviously zero and the spectral radius of $B_{\epsilon, \eta}$ is also null. This means that the algorithm converges in a single iteration because the two stages are uncoupled: the second stage is the condensed problem on the interface of the substructured problem. This result only underlines the consistency and the substantial enhancement of the second version of the method for a non-zero $\epsilon$ parameter. The spectral radius may be evaluated in the examples for each value of $\eta$, with $\epsilon$ being fixed at 1 or 0 in Figure 6 . However for the asymptotic case, $\epsilon=0$, the optimal zero value of the spectral radius does not seem to be reached, especially when the size of the system increases from example one to the third example.

\section{Asymptotic analysis}

Such an analysis concerns two limit-case situations. The second one, the nonsmooth case, is the final focus of this paper. It consists of determining the contact impulses and induced velocities at a given instant in a dense granulate impacted by particles. We limit ourselves here 


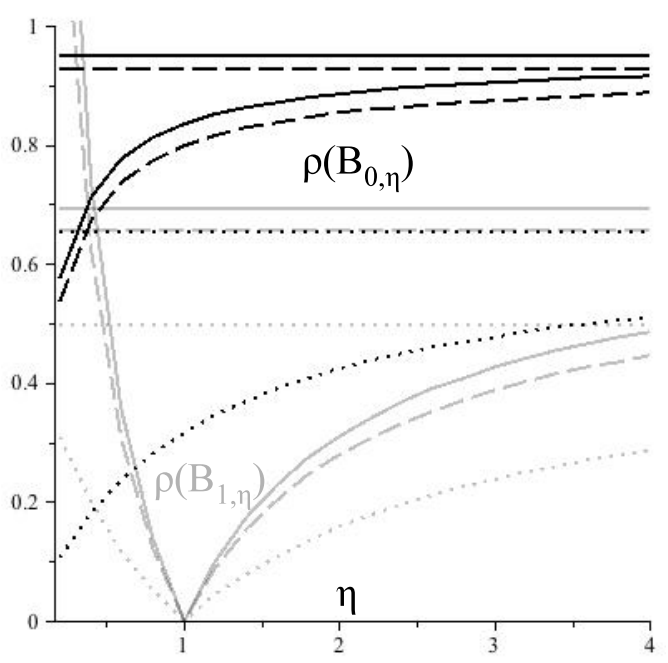

Figure 6: Spectral radius vs. $\eta$, for $\epsilon=1$ and for $\epsilon=0$ and the three examples: 4 disks (dot), 8 disks (dash), 12 disks (solid)

to the analysis of the frictionless contact case (this case is called nonsmooth in the following). The first situation is not related to a physical realistic case, but is an intermediate problem viewed as the limit case of the linear smooth problem where the flexibility parameter $\epsilon$ tends to zero. We then have to determine, in this linear problem, a distribution of impulses between rigid bodies without possible contact loss. This is similar to the determination of tensions in an hyperstatic network of rigid bars.

\subsection{Rigid limit case with the generic algorithm}

When $\epsilon$ tends to zero, the limit contraction matrix is then equal to:

$$
B_{0}=X^{-1} Y_{0} \quad \text { where } \quad Y_{0}=\sum_{E=1}^{n_{s d}} \hat{A}_{E \Gamma} W_{E}^{-1} \hat{A}_{E \Gamma}^{T}
$$

It is not surprising that the spectral radius increases when parameter $\epsilon$ tends to zero as shown in Figure 5. Moreover, this matrix is not always evaluable if the Delassus operator $W_{E}$ is not invertible, i.e. when the contact network is hyperstatic. This is a common situation in dense granulates generating the non-uniqueness of the contact impulse distribution.

\subsection{Rigid limit case with the enriched algorithm}

If we plot the spectral radius of the contraction matrix $\rho\left(B_{0, \eta}\right)$ with respect to $\eta$, as in Figure 6, we obtain a decreasing value of the spectral radius when $\eta$ tends to zero, but a null spectral radius does not seem to be reachable. To directly verify the consistency of the limit problem, $\eta=0$ for $\epsilon=0$, we have to handle an approximation of the matrix $M_{E, \eta}^{-1}$ when $\eta$ tends to zero,

$$
M_{E, \eta}^{-1}=\left(M_{E}+\frac{1}{\eta} K_{E}\right)^{-1} \approx M_{E}^{\infty}+\eta K_{E}^{+}
$$


where the null space of $M_{E}^{\infty}$ contains the range of $K_{E}$ (or equivalently of $H_{E}$, i.e. $M_{E}^{\infty} H_{E}=0$ ) and $K_{E}^{+}$is the Moore-Penrose pseudo-inverse of $K_{E}$ modulo the mass matrix $M_{E}$. At this point, we can rewrite the second stage of the enriched algorithm when $\eta$ tends to zero, and for any local constitutive law (even for nonlinear or nonsmooth laws):

$$
X^{\infty} \bar{F}_{\Gamma}=\sum_{E=1}^{n_{s d}} A_{E \Gamma}\left[M_{E}^{\infty} F_{E}^{d}+K_{E}^{+} H_{E} \bar{v}_{E}\right] \quad \text { with } \quad X^{\infty}=\sum_{E=1}^{n_{s d}} A_{E \Gamma} M_{E}^{\infty} A_{E \Gamma}^{T}
$$

The linear rigid limit case is characterized by a kinematic material variable field $\bar{v}_{E}$ (relative velocities between grains for granular systems) equal to zero, so that both stages in the algorithm are decoupled, and the convergence requires a single iteration, as in the linear smooth case. It is, however, interesting to note that the mapping $M_{E}^{\infty}$ is non zero only on the null space of $K_{E}$, that means on the rigid body motions of subdomain $E$. The elimination of such a subspace is the key ingredient of the Domain Decomposition preconditioners in elastostatics and even in elastodynamics of continuum media.

\subsection{Nonsmooth case with the generic algorithm}

A first attempt to analyze convergence for a nonsmooth case involves considering a complementarity condition between $r_{E}$ and $v_{E}$ (characteristic of frictionless unilateral between-grain contact in granulates). Such a condition may be interpreted as a piecewise linear relationship. The global convergence is difficult to analyze $[25,20]$ but we may study the local convergence as performed, for instance, by [26]. We then suppose that the solution is characterized by a rigid limit case given by a compatibility operator $G_{E}$ that differs from the initial $H_{E}$. This compatibility operator $G_{E}$ corresponds to the only active contacts which cannot be determined a priori in a general case; this stage requires a specific algorithm as the NonLinear Gauss-Seidel method for instance. However, for the simple examples studied here it is possible to postulate the active contacts by considering the only compressive links (black lines on the resulting tension networks of the Figures 2, 3 and 4) obtained with the linear modeling. The generic algorithm then behaves, near the solution, like a new algorithm where the Stage 1 uses the "local" compatibility condition given by $G_{E}$ while the Stage 2 uses the "global" compatibility condition given by $H_{E}$. More precisely, we consider the following relation:

$$
R_{E}=H_{E} r_{E}=G_{E} \bar{r}_{E}
$$

then, $r_{E}=C_{E} \bar{r}_{E}$ and $H_{E} C_{E}=G_{E}$.

The first stage of the generic algorithm 1 is modified as follows: $G_{E}$ replaces $H_{E}$ in $W_{E}, \hat{A}_{E \Gamma}$ and $\hat{f}_{\Gamma}$ to define $\bar{W}_{E}, \bar{A}_{E \Gamma}$ and $\bar{f}_{\Gamma}\left(\bar{A}_{E \Gamma}^{T}=G_{E}^{T} M_{E}^{-1} A_{E \Gamma}^{T}, \bar{W}_{E}=G_{E}^{T} M_{E}^{-1} G_{E}\right.$, recall in this case $\bar{v}_{E}$ is equal to zero)

$$
\text { Stage 1: } \quad \bar{W}_{E} \bar{r}_{E}=-\bar{v}_{E}^{d}+\bar{A}_{E \Gamma}^{T} \bar{F}_{\Gamma}
$$

The second stage is only slightly modified: $\bar{r}_{E}$ is replaced by $C_{E} \bar{r}_{E}$ :

$$
\text { Stage 2: } \quad X \bar{F}_{\Gamma}=\hat{f}+\sum_{E=1}^{n_{s d}} \hat{A}_{E \Gamma} C_{E} \bar{r}_{E}=\hat{f}+\sum_{E=1}^{n_{s d}} \bar{A}_{E \Gamma} \bar{r}_{E}
$$

The limit contraction matrix corresponds to the one associated with the limit rigid linear case, while using $G_{E}$ instead of $H_{E}$. For the three examples, the spectral radius is represented in 
Figure 7 with a horizontal line:

$$
\bar{B}=X^{-1} \bar{Y} \quad \text { where } \quad \bar{Y}=\sum_{E=1}^{n_{s d}} \bar{A}_{E \Gamma} \bar{W}_{E}^{-1} \bar{A}_{E \Gamma}^{T}
$$

\subsection{Nonsmooth case with the enriched algorithm}

The expression of the new search direction has to be rewritten for local analysis of the convergence of the enriched algorithm because $\tilde{v}_{E}$ and $\tilde{r}_{E}$ are defined with $H_{E}$ whereas $\bar{v}_{E}$ and $\bar{r}_{E}$ are defined with $G_{E}$ :

$$
\left(\tilde{r}_{E}-\bar{r}_{E}\right)+\frac{1}{\eta}\left(\tilde{v}_{E}-\bar{v}_{E}\right)=0 \quad \Longrightarrow \quad\left(\tilde{r}_{E}-C_{E} \bar{r}_{E}\right)+\frac{1}{\eta}\left(\tilde{v}_{E}-H_{E}^{T} \bar{V}_{E}\right)=0
$$

Consequently, only the second stage of the algorithm is modified as follows:

$$
\text { Stage 2: } \quad X_{\eta} \bar{F}_{\Gamma}=\sum_{E=1}^{n_{s d}} A_{E \Gamma} M_{E, \eta}^{-1}\left[F_{E}^{d}+H_{E} C_{E} \bar{r}_{E}+\frac{1}{\eta} K_{E} \bar{V}_{E}\right]
$$

The contraction matrix depends on the numerical flexibility parameter $\eta$ :

$$
\bar{B}_{\eta}=X_{\eta}^{-1} \bar{Y}_{\eta}
$$

where

$$
\begin{aligned}
\bar{Y}_{\eta} & =\sum_{E=1}^{n_{s d}} A_{E \Gamma} M_{E, \eta}^{-1} H_{E} Z_{E, \eta} A_{E \Gamma}^{T} \\
Z_{E, \eta} & =C_{E} \bar{W}_{E}^{-1} G_{E}^{T} M_{E}^{-1}+\frac{1}{\eta}\left(H_{E}^{T} M_{E}^{-1} G_{E} \bar{W}_{E}^{-1} G_{E} M_{E}^{-1}-H_{E}^{T} M_{E}^{-1}\right)
\end{aligned}
$$

Such an operator is quite difficult to analyze from a theoretical standpoint. Nevertheless, it can be tested on the examples, by determining the spectral radius with respect to parameter $\eta$.

We note in Figure 7 that the spectral radius may be smaller than that of the generic algorithm, get closer to this value when $\eta$ increases, but get larger than 1 when $\eta$ tends to zero. However, greater the number of disks, the more the optimal range of $\eta$ is close to zero and reduced. Such behavior seems to exclude the use of the asymptotic version $(\eta=0)$ to solve nonsmooth dynamic systems as granular ones. However, such a theoretical asymptotic analysis exemplifies the consistency of the previous approach and opens numerical perspectives. Indeed, when $\eta$ tends to zero, the contraction matrix takes the following form:

$$
\bar{B}^{\infty}=X^{\infty} \bar{Y}^{\infty} \quad \text { where } \quad \bar{Y}^{\infty}=\sum_{E=1}^{n_{s d}} A_{E \Gamma} K_{E}^{+} H_{E} Z_{E}^{\infty} A_{E \Gamma}^{T}
$$

The $Z_{E}^{\infty}$ operator vanishes when $G_{E}=H_{E}$ according to the result of Section 4.2:

$$
Z_{E}^{\infty}=\underbrace{H_{E}^{T} M_{E}^{-1} G_{E}}_{\bar{W}_{E} \text { if } G_{E}=H_{E}} \bar{W}_{E}^{-1} G_{E} M_{E}^{-1}-H_{E}^{T} M_{E}^{-1}=0 \text { if } G_{E}=H_{E}
$$

The two stages are decoupled: the second one computes the interface multipliers $F_{\Gamma}$ and the first one determines the internal material variables $v_{E}$ and $r_{E}$. This result may be extended to a 


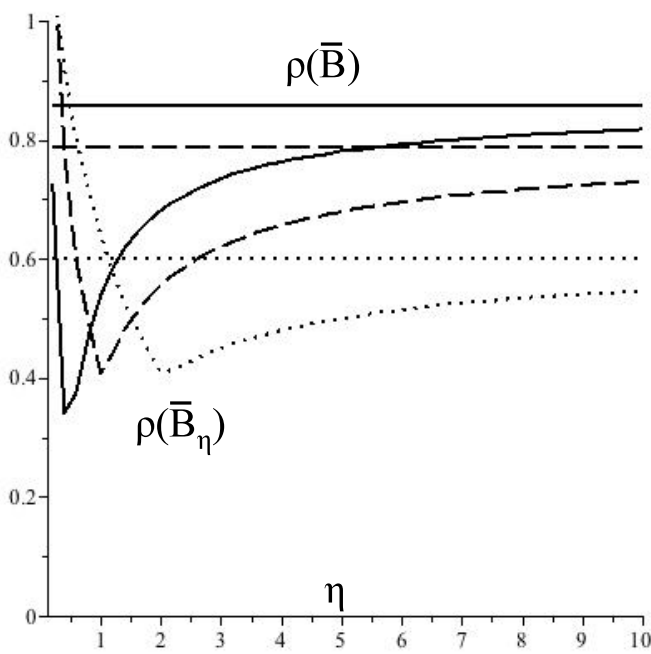

Figure 7: Spectral radius with respect to $\eta$ for the three examples: 4 disks (dot), 8 disks (dash), 12 disks (solid)

more general situation where the rank of $H_{E}$ is less than that of $G_{E}$. If we refer to a granular type problem, this case means that the a priori potential contacts considered at the global stage are less numerous than the active ones. In this case, the two stages are still decoupled but the solution does not satisfy all the equations because the second global stage does not take all active links (contacts) represented by the $G_{E}$ mapping into account. The numerical strategy presented in the conclusion is based on this feature.

\section{Numerical granular tests}

The previous algorithms have been implemented in the development platform LMGC90 ${ }^{1}$. The first example concerns a large-scale two dimensional shear test with disks as grains and frictional contact as interaction model. The friction coefficient is $\mu=0.3$, and 100000 grains have been packed under gravity to get the sample of Figure 8. The right and left boundaries have a prescribed rotating motion. The box is split vertically into two subdomains.

The generic algorithm 1 is used and two convergence indicators are computed to control the iterations. Indeed, no reference error is available due to the non-uniqueness of the solution. The volume relative error indicator is computed on each interaction, and summed up over the whole domain, without reference to any substructuring. It is defined at iteration $i$ as:

$$
\zeta_{i}=\sqrt{\frac{\left(r_{i}-r_{i-1}\right)^{T}\left(r_{i}-r_{i-1}\right)}{r_{i}^{T} r_{i}}}
$$

The second error indicator is defined only on the interfaces, and measures the stationarity of the

${ }^{1}$ http://www.lmgc.univ-montp2.fr/LMGC90/ 
interforces:

$$
Z_{i}=\sqrt{\frac{\left(F_{\Gamma, i}-F_{\Gamma, i-1}\right)^{T}\left(F_{\Gamma, i}-F_{\Gamma, i-1}\right)}{F_{\Gamma, i}^{T} F_{\Gamma, i}}}
$$

Their evolutions along iterations are depicted on Figure 9. A large number of iterations is required for underlining the different behavior of the two indicators since the difference appears essentially after 1000 iterations. However, such a large number may be also useful for simulating precisely enough unstable behaviors of granular structures involving for instance shear bands.

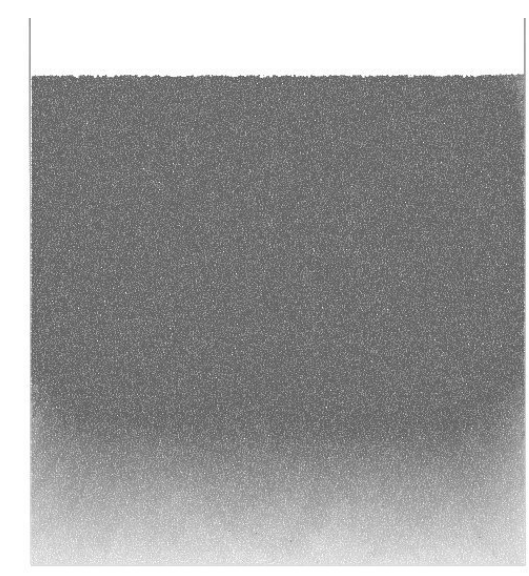

Figure 8: Two dimensional shear test on 100000 disks with friction

These evolutions are quite similar, and the second indicator is selected in the following, since it is less costly.

In a second step, the generic and enriched versions of the proposed algorithm are tested on a smaller shear example with a free top side split into two subdomains. The initial and final configurations of this test are drawn in Figure 10. The full process is simulated with 250 time steps.

For each version, two numbers of Gauss-Seidel iterations per subdomain are tested: $n_{\mathrm{GS}}=$ 1 and $n_{\mathrm{GS}}=100$, see Figure 11. Roughly, the convergence rates are similar, with a minor improvement for the enriched version. More precisely, for $n_{\mathrm{GS}}=1$, the gain is more marked during the first iterations, and vanishes asymptotically, when the majority of corrections are local within the subdomains, and mainly concern the weak force network [4]. For $n_{\mathrm{GS}}=100$, less improvement is observed and the asymptotic convergence rates are identical, and obviously better than for $n_{\mathrm{GS}}=1$.

The scalability of the strategy is not improved by the enriched version because it is in fact satisfied with the generic algorithm as discussed in a previous work [23]. As analyzed in this paper, it could be attributed to the rusticity of the nonlinear solver based on a simple fixed point technique. But the objective of the enriched version is not to recover the scalability but to have the ability to switch if needed from a nonsmooth discrete granular microscopic model to a (preferentially linear) continuous macroscopic model. The enrichment is a first step in this way.

The speed-up is not given here due to some implementation burdens: memory duplications and non efficient memory copy operations in the current code that are large enough to get non 


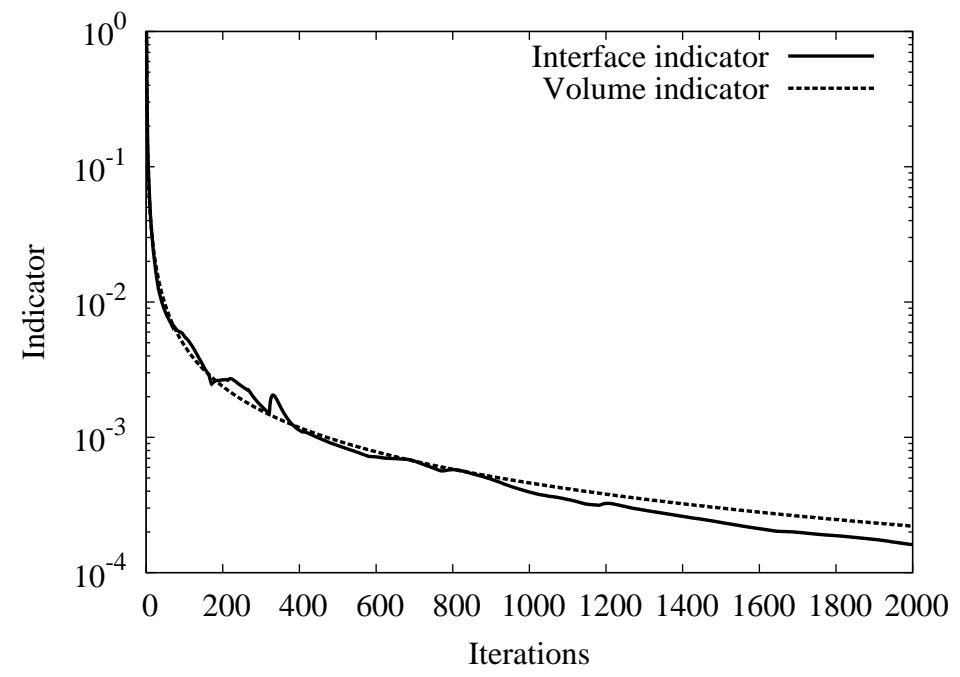

Figure 9: Comparison of the interface and volume indicator on a granular case with 100000 grains
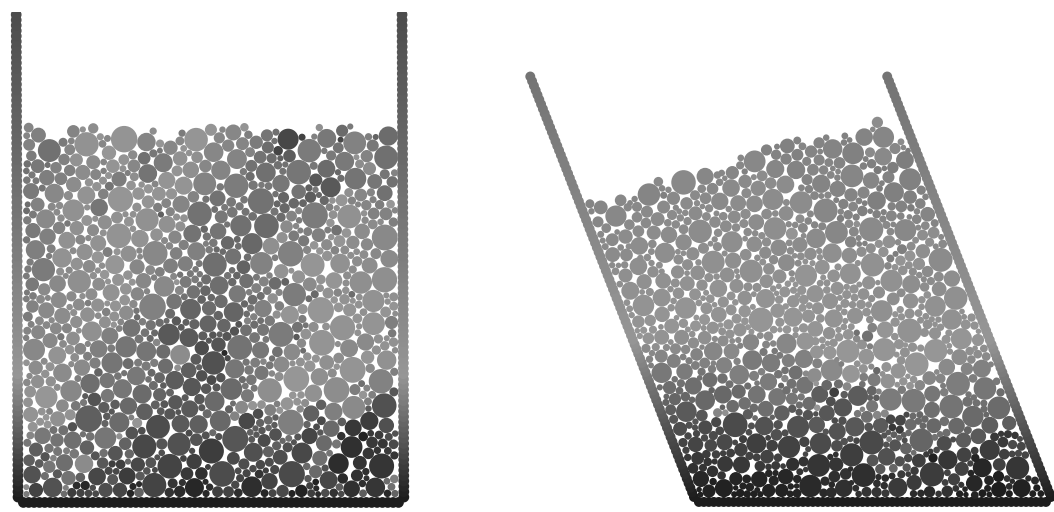

Figure 10: Two dimensional shear test on 1000 disks with friction

pertinent time measures. Improving implementation to allow effective CPU costs reporting is under work.

\section{Conclusions}

The domain decomposition strategy proposed in this article for the numerical simulation of nonsmooth large-scale dynamic systems is inspired by the latest developments used for regular 


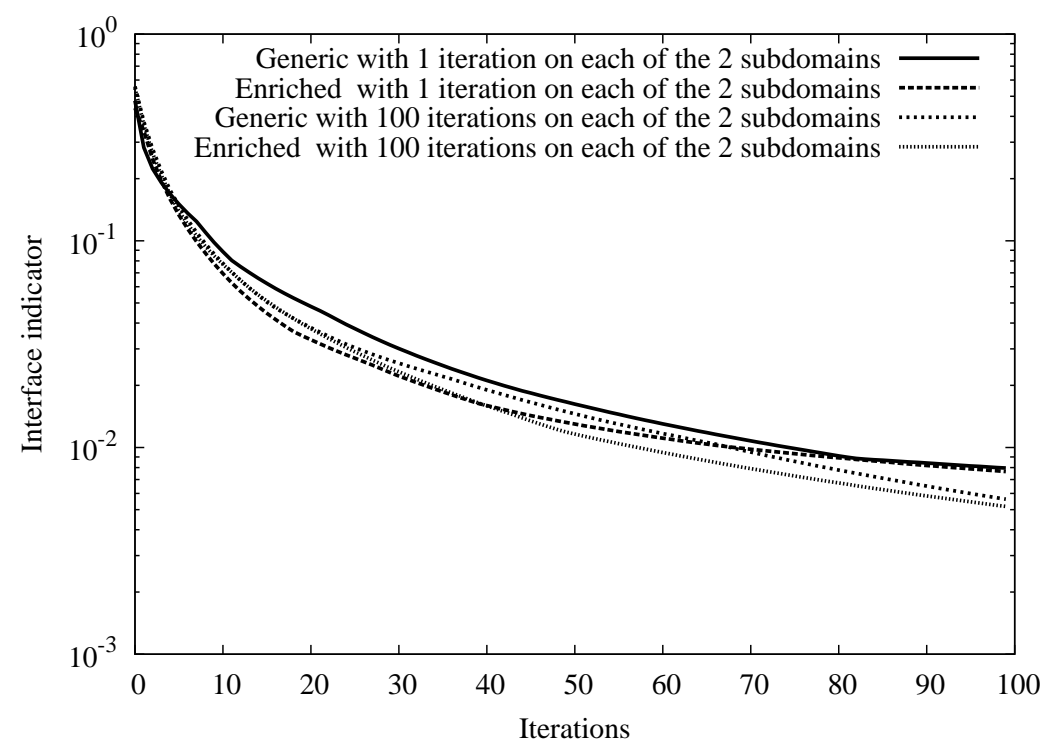

Figure 11: Comparison of classical and enriched algorithms on a granular case with 1000 grains

continuum media. The enhanced version refers to the concept of spatial multilevel preconditioners with a coarse problem to improve convergence. This first study, with analytical and numerical aspects, provides mixed results. Indeed, for the concerned topic, the cost of this enhancement is too high for the convergence gain it provides. As exemplified by "analytical" cases, and numerical investigations, this result could be explained by: (i) the rigid nature of the particles in dynamic evolution, and (ii) the nonsmooth character of the interactions.

Therefore, such an enhanced approach could not be used as it is, but may lead to a semiimplicit solution by getting rid of iterations between the two stages. As mentioned at the end of Section 4.4, the network underlying the mapping $H_{E}$ may be chosen differently for the two stages of the algorithm. This network can be frozen during the linear Stage 2 using the previous time step configuration. This numerical strategy is perhaps justified by a space-time multiscale behavior of granular systems, but this remains to be investigated in future studies.

\section{References}

[1] M. Jean, The non-smooth contact dynamics method, Computer Methods in Applied Mechanics and Engineering 177 (1999) 235-257.

[2] J. J. Moreau, Numerical aspects of sweeping process, Computer Methods in Applied Mechanics and Engineering 177 (1999) 329-349.

[3] P. A. Cundall, O. D. L. Strack, A discrete numerical model for granular assemblies, Geotechnique 29 (1) (1979) 47-65.

[4] F. Radjai, D. E. Wolf, M. Jean, J. J. Moreau, Bimodal character of stress transmission in granular packings, Physical Review Letters 80 (1) (1998) 61-64.

[5] G. Saussine, C. Cholet, P.-E. Gautier, F. Dubois, C. Bohatier, J. J. Moreau, Modelling ballast behaviour under dynamic loading, part 1: A 2D polygonal discrete element method approach, Computer Methods in Applied Mechanics and Engineering 195 (19-22) (2005) 2841-2859. 
[6] A. Rafiee, M. Vinches, C. Bohatier, Modelling and analysis of the Nîmes arena and the Arles aqueduct subjected to a seismic loading, using the Non-Smooth Contact Dynamics method, Engineering Structures 30 (12) (2008) 3457-3467.

[7] P. Le Tallec, Domain-decomposition methods in computational mechanics, Computational Mechanics Advances 1 (2) (1994) 121-220, North-Holland.

[8] C. Farhat, A Lagrange multiplier based divide and conquer finite element algorithm, Journal of Computing Systems in Engineering 2 (1991) 149-156.

[9] J. Mandel, Balancing domain decomposition, Communications in Applied Numerical Methods 9 (1993) $233-241$.

[10] C. Farhat, M. Lesoinne, K. Pierson, A scalable dual-primal domain decomposition method, Numerical Linear Algebra with Applications 7 (2000) 687-714.

[11] J. Mandel, R. Tezaur, C. Farhat, A scalable substructuring method by Lagrange multipliers for plate bending problems, SIAM Journal on Numerical Analysis 36 (5) (1999) 1370-1391.

[12] C. Farhat, P. S. Chen, J. Mandel, A scalable Lagrange multiplier based domain decomposition method for timedependent problems, International Journal for Numerical Methods in Engineering 38 (22) (1995) 3831-3854.

[13] S. Nineb, P. Alart, D. Dureisseix, Domain decomposition approach for nonsmooth discrete problems, example of a tensegrity structure, Computers and Structures 85 (9) (2007) 499-511.

[14] Y.-H. De Roeck, P. Le Tallec, M. Vidrascu, A domain decomposed solver for nonlinear elasticity, Computer Methods in Applied Mechanics and Engineering 99 (1992) 187-207.

[15] M. Barboteu, P. Alart, M. Vidrascu, A domain decomposition strategy for nonclassical frictional multi-contact problems, Computer Methods in Applied Mechanics and Engineering 190 (2001) 4785-4803.

[16] P. Gosselet, C. Rey, Non-overlapping domain decomposition methods in structural mechanics, Archives of Computational Methods in Engineering 13 (4) (2006) 515-572.

[17] D. Dureisseix, C. Farhat, A numerically scalable domain decomposition method for the solution of frictionless contact problems, International Journal for Numerical Methods in Engineering 50 (12) (2001) 2643-2666.

[18] Z. Dostál, D. Horák, Scalable FETI with optimal dual penalty for a variational inequality, Numerical Linear Algebra with Applications 11 (5-6) (2004) 455-472.

[19] Z. Dostál, D. Horák, R. Kucera, V. Vondrák, J. Haslinger, J. Dobiás, S. Pták, FETI based algorithms for contact problems: scalability, large displacements and 3d coulomb friction, Computer Methods in Applied Mechanics and Engineering 194 (2-5) (2005) 395-409.

[20] F. Jourdan, P. Alart, M. Jean, A Gauss-Seidel like algorithm to solve frictional contact problems, Computer Methods in Applied Mechanics and Engineering 155 (1-2) (1998) 31-47.

[21] P. Ladevèze, A. Nouy, O. Loiseau, A multiscale computational approach for contact problems, Computer Methods in Applied Mechanics and Engineering 191 (2002) 4869-4891.

[22] P. Ladevèze, Nonlinear computational structural mechanics — New approaches and non-incremental methods of calculation, Springer Verlag, 1999.

[23] D. Iceta, D. Dureisseix, P. Alart, Mixed versus impulse-oriented domain decomposition method for granular dynamics, European Journal of Computational Mechanics 18 (5-6) (2009) 429-443.

[24] J. A. C. Martins, A. P. da Costa, Stability of finite-dimensional nonlinear elastic systems with unilateral contact and friction, International Journal of Solids and Structures 37 (18) (2000) 2519 - 2564

[25] P. Alart, Méthode de Newton généralisée en mécanique du contact, Journal de Mathématiques Pures et Appliquées 76 (1) (1997) 83-108.

[26] P. W. Christensen, A. Klarbring, J. S. Pang, N. Strömberg, Formulation and comparison of algorithms for frictional contact problems, International Journal for Numerical Methods in Engineering 42 (1998) 145-173. 